\title{
La representación cartográfica del paisaje cultural y el patrimonio inmaterial
}

José María Rodrigo Cámara, José Manuel Díaz Iglesias, Laboratorio del Paisaje, Centro de Documentación y Estudios, IAPH

\section{EL PAISAJE Y EL PATRIMONIO INMATERIAL COMO RETOS CARTOGRÁFICOS: DE LAS PERCEPCIONES Y LAS IDEAS AL MAPA}

La evolución en los últimos años de los conceptos de cultura y patrimonio ha proporcionado "nuevos objetos", quizás -por el momento- más complejos, por ser holísticos y transversales respecto a campos disciplinares antes cerrados en si mismos, que manifiestan una relación diferente con la medida del tiempo histórico y que se encuentran muy cercanos a dinámicas antes menos tratadas, como son la de su propia construcción social o la de su pertenencia al territorio en el que se producen. Patrimonio inmaterial y paisajes ejemplifican este nuevo carácter temporal, social y territorial:

- Lejos de ser un patrimonio "estático", son un patrimonio "vivo y actual" porque, aún manteniendo profundidad en el tiempo histórico, viven e incluso necesitan estar y evolucionar en el presente.

- En estos nuevos objetos patrimoniales, su formulación, identificación y gestión se asocian más a lo colectivo y participativo, en un proceso que se encuentra en ocasiones en plena crisis, debate, critica, interpretación y/o actualización en el seno de la sociedad a la que pertenecen.

- Estas nuevas experiencias de lo cultural y patrimonial, por último, necesitan un espacio-lugar en el que reconocerse. Su profunda territorialidad les hace ser muy sensibles a los cambios que se produzcan en su territorio lo que en situaciones extremas puede hacer peligrar hasta su propia continuidad como fenómeno cultural (GALINDO GONZÁLEZ; SABATÉ BEL, 2009).

Si hoy tratamos con nuevos elementos del patrimonio, altamente codificados a tres niveles y que pueden ser tratados también como parte de un problema de símbolos y mensajes, es decir, de semiología, nos debemos preguntar si hemos actualizado la teoría y el método cartográfico para representarlos como objetos que hay que identificar y descomponer (decodificar/leer) para luego interpretar y representar (objetivar/comunicar).

\section{Un marco teórico}

Una vez superados los modelos, primero descriptivos, sistémicofuncionalistas y positivistas de los años sesenta y setenta, que se englobaron en la llamada "nueva geografia", actualmente se con- sidera a la geografía cultural, más concretamente su actual lectura desde los años noventa sobre bases teóricas establecidas ya a finales del siglo XIX, como el enfoque más activo de la moderna Geografía, tanto en el ámbito anglosajón como en el que podría denominarse "latino", encabezado por Francia.

La nueva teoría geográfica va inseparablemente unida a la evolución de los conceptos de cultura, espacio y sociedad. Autores como Paul Claval explican cómo en aquella primera concepción de la geografía cultural se abordaban los estudios exclusivamente "desde fuera", como "naturalistas culturales", a través de sus artefactos, construcciones y encuadres paisajísticos (CLAVAL, 1999: 26). Más tarde se pasó a reivindicar el papel de la cultura entendida como estructura de comunicación en el que el flujo de ideas y acciones sobre el mundo real consiste en informaciones a través de la observación, la imitación y la palabra, que no dejan de fluir entre el individuo, el grupo, la comunidad... Ésta es la "visión del mundo" y, por tanto, de la Geografía y la cultura.

Este tratamiento del "espacio geográfico" es plenamente cultural. No resulta extraño que la etnogeografía, como "toma en consideración del saber geográfico de los grupos" (CLAVAL, 1999: 35; COSGROVE; DANIELS, 1988), y el paisaje, como "impronta y matriz de la cultura" (BERQUE, 1984), se hayan convertido recientemente en campos de interés principal para el trabajo ya no sólo de los geógrafos, sino también de arqueólogos e historiadores, de antropólogos y sociólogos, de arquitectos y diseñadores, incluso de filósofos o de gestores culturales, económicos, políticos, etc.

En este contexto, la Cartografía sería la herramienta o el vehículo de construcción de imágenes o representaciones de ese "espacio geográfico" una vez pasado por el tamiz de la cultura y el grupo social mediante una suerte de "lenguaje" válido, consensuado o convencional, tanto para la codificación del discurso como para la decodificación del mensaje. En esta visión, el documento cartográfico es un soporte de discurso plenamente postmoderno tal como es aplicado en la teoría del arte, la estética, del diseño o de la comunicación, y asi puede ser proyectado sobre los conceptos de icono, símbolo, imagen y mensaje.

La cartografía postmoderna, por tanto, es un concepto susceptible de ser deconstruido (decodificado) y que es reflejo de cómo 
la sociedad es productora de espacio geográfico entendido como algo diferente del espacio físico (HARLEY, 1989; LEFEBVRE, 1991):

- Es un medio de investigación-conocimiento porque puede integrar no sólo la descripción formal o retórica del espacio geográfi$\mathrm{Co}$, sino también su vertiente metafórica, como la inmaterialidad en cuanto a significados simbólicos hipercodificados o su perceptibilidad como paisaje siempre sujeta al marco cultural y social.

- Es un medio de proyección para la gestión-creación del espacio social porque procura ser proactivamente político.

- Es un medio de comunicación porque es mensaje que traduce cómo es la idea de espacio de cada grupo o comunidad, y este mensaje puede ofrecerse desde lo informativo, lo educativo, lo publicitario, hasta lo plenamente político (HARLEY, 1989) o lo demagógico, etc.

\section{Paisaje e inmaterialidad en el campo cartográfico}

Las nuevas representaciones cartográficas del patrimonio inmaterial y el paisaje van a tener referentes en líneas de experimentación tan interesantes como las conocidas como atlas de comunidades indigenas desarrolladas desde iniciativas de la Geografia, la Antropologia y la propia sociedad civil (HIRT, 2006; HERLIHY; DOBSON; AGUILAR et ál., 2008; CHAPIN; THRELKELD, 2008; SLETTO, 2009), empleando aproximaciones metodológicas basadas en la elaboración de mapas colaborativos por las propias poblaciones que luego son trasladados a matrices cartográficas convencionales. Las representaciones del espacio funcional de producción, residencial, simbólico, ritual, mágico, político, etc., son así tratadas para permitir la mayor reducción posible de rasgos de etnocentrismo o para reflejar la participación de los diversos agentes de la sociedad civil (WU; ISAKSSON, 2008). En otros casos se producen ensayos de formalización de nuevas categorias de representación menos usuales como las que caben en la órbita de lo anímico o lo religioso, o las perspectivas de género, de poder, de marginalidad, etc.

Si estas experiencias están abriendo nuevas vias que aproximan el compromiso entre el concepto de sociedad-cultura y la representación de sus espacios sobre mapas, debe señalarse igualmente el gran desarrollo de una tradición cartográfica más vinculada a la gestión pública territorial. En este último caso podrá observarse cómo van a generarse mapas cuya finalidad es la precisión y la georreferenciación de los temas, con atención a los límites de cada unidad de paisaje y a sus relaciones con otras entidades representativas en el territorio, en tanto que el producto va a orientarse a la planificación-ordenación en diferentes escalas espaciales o al tratamiento administrativo del sistema de protección.

Como heredera de su propia tradición nacional de la Geografia regional clásica que recoge los trabajos desde Vidal de la Blache, puede citarse, a nivel de paisaje, la trayectoria, desde hace más de quince años, de una línea coherente de productos como los atlas de paisaje departamentales en Francia. Ya desde 1994 se documenta un primer esfuerzo en la normalización de la elaboración de los atlas que tendrá continuidad hasta nuestros días (LUGINBÜHL, 1994; BRUNET-VINCK, 2004; SEGUIN, 2009; VESCHAMBRE, 2009). Su elaboración parte de un análisis combinado, tanto desde la morfología física y la Ecología, como desde la estructura territorial de líneas y redes de comunicación o del poblamiento. Se trata, en sintesis, de lograr la diferenciación analítica de unidades de paisaje y extraer su carácter paisajístico (ROCHE, 2007; 2009), un proceso que constituirá la base del discurso empleado en los diferentes atlas. Es destacable cómo esta tradición también impregna la evolución de los catálogos de paisaje de Cataluña como puede verse en documentos programáticos (NOGUÉ; SALA, 2006: 38) y en los catálogos ya publicados (NOGUÉ; SALA, 2010).

En el ámbito anglosajón, es destacable la normalización cartográfica impulsada por los Landscape Character Assessment (LCA) gestionados por el organismo público Natural England (LANDSCAPE, 2002) y la atención prestada al patrimonio cultural mediante los Historic Landscape Characterisation (HLC) (CLARK; DARLINGTON; FAIRCLOUGH, 2004) realizados por English Heritage. En este caso los aspectos de percepción de la historia en los paisajes y su intento de representación cartográfica tienen aqui uno de sus mejores exponentes desde el trabajo de una administración pública. Estas experiencias han generado nuevos desarrollos cartográficos que vinculan el paisaje y sus elementos patrimoniales con otro tipo de información basada en los aspectos más sensoriales de la "experiencia" percibida in situ.

Pueden citarse los trabajos del Countryside Council de Gales en el marco de LANDMAP (http://landmap.ccw.gov.uk/methodology/), entre los que se cuenta la elaboración de una metodología de registro para los aspectos sensoriales y participativos, como los mapas de sonido, de sensibilidad visual, de aspectos sociales o etnopaisajísticos - los cuales son denominados cultural landscapes y están basados en la relación entre las personas y los lugares- diferenciándolos, por tanto, de su otra categoria, historic landscapes, más vinculados con la remanencia en el territorio de inmuebles y de otros elementos de infraestructura a lo largo del tiempo (usos, propiedad, comunicaciones, transporte, asentamientos).

\section{CARTOGRAFÍA EN EL IAPH SOBRE NUEVOS TEMAS PATRIMONIALES}

El trabajo continuo con entidades patrimoniales consideradas en su espacio territorial es una realidad de larga tradición en el IAPH, constatable desde el momento en el que se inician líneas de investigación y desarrollo del registro y documentación digital del patrimonio histórico andaluz, comenzando por las entidades arqueológicas y continuando en el presente con el patrimonio contemporáneo, el inmaterial o el paisaje. Estas aproximaciones han generado experiencias como el Atlas del Patrimonio Inmaterial de Andalucia actualmente en curso de realización y en el que ya se apunta claramente en sus planteamientos metodológicos el dise- 


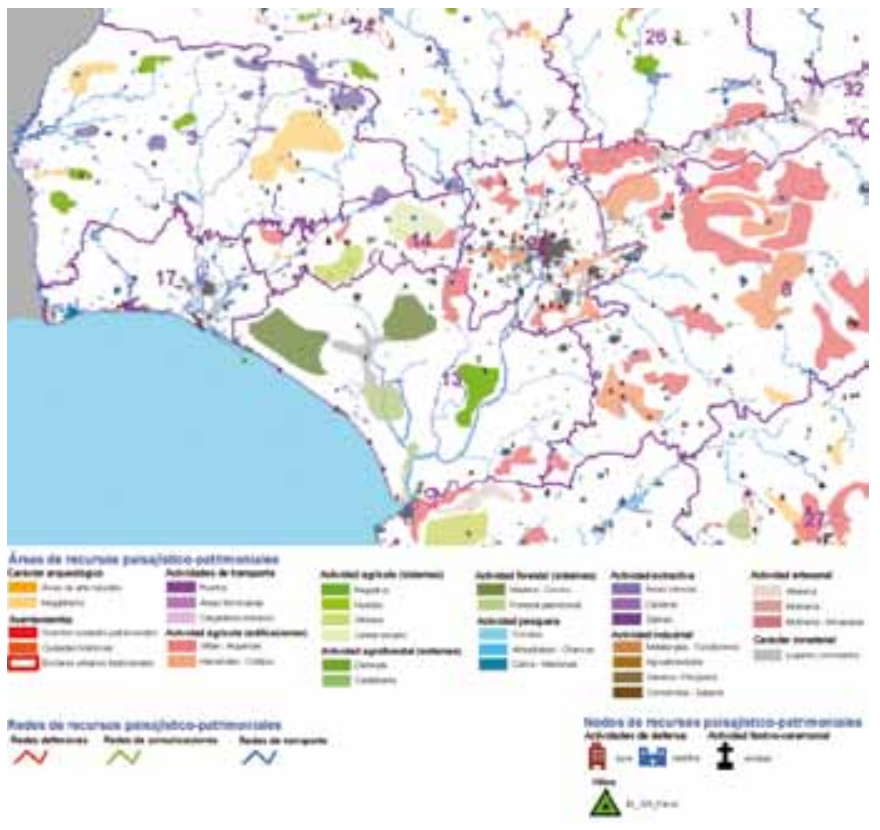

Proyecto TRAZA. Detalle de cartografía de paisaje y recursos patrimoniales con estructura en áreas, redes y nodos. Fuente: Laboratorio del Paisaje, IAPH

ño de "un modelo conceptual para la representación geoespacial del patrimonio inmaterial, adaptado a sus caracteristicas especificas donde el elemento geográfico está sujeto a su valor simbólico y a un marco temporal concreto" (CARRERA DÍAZ, 2009: 21). En este caso, serán necesarios unos criterios para el geo-registro de de los temas toda vez que los elementos materiales existentes servirán de apoyo material para construir la cartografía de cada manifestación de patrimonio inmaterial (QUINTERO MORÓN, 2003; CARRERA DÍAZ, 2009: 28).

En el marco de las becas de investigación convocadas por el IAPH se desarrolló durante 2004-2005 el proyecto denominado Representación cartográfica de los paisajes (CHICLANA RODRÍGUEZ, 2005) en el que se aportaban un estudio conceptual y dos aplicaciones de campo sobre ejemplos de marcado carácter patrimonial. Se llevaba a cabo una metodología de valoración paisajistica mediante el uso intensivo de las tecnologías de información geográfica, logrando finalmente un trabajo eminentemente práctico sobre cuestiones de actuaciones sobre el paisaje (CHICLANA RODRIGUEZ; FERNÁNDEZ CACHO, 2005).

Más recientemente, el paisaje cultural a escala regional ha sido tratado por el IAPH en el marco de un proyecto que tenía como objetivo la caracterización patrimonial del mapa de paisajes de Andalucia (FERNÁNDEZ CACHO; FERNÁNDEZ SALINAS; HERNÁNDEZ LEÓN et ál., 2008). El mapa de paisajes regional había sido publicado en 2005 dentro de la serie del Atlas de Andalucía (tomo II) y en su escala de elaboración se utilizaron básicamente la morfologia, usos y coberturas vegetales para construir una serie de áreas y ámbitos de paisaje que se combinan en un conjunto amplio de unidades fisionómicas dando lugar a 422 paisajes diferenciados.
Faltaba, por tanto, una lectura de la profundidad cultural y patrimonial en el tiempo, en las actividades y en las visiones locales y foráneas que aún pueden rastrearse en los paisajes andaluces (FERNÁNDEZ CACHO; FERNÁNDEZ SALINAS; HERNÁNDEZ LEÓN et ál., 2008). Los resultados de este proyecto se materializaron recientemente (FERNÁNDEZ CACHO; FERNÁNDEZ SALINAS; HERNÁNDEZ LEÓN et ál., 2010) ofreciendo un apoyo cartográfico para las 32 demarcaciones de paisaje cultural que sirvieron como estructura para el análisis regional en donde se ha tratado de sintetizar procesos históricos y actividades con los referentes materiales de patrimonio construido, sistemas agrarios, infraestructuras, etc. con valores de legado cultural en el territorio y de elemento generador de los paisajes regionales.

Como continuación de esta misma línea se está trabajando actualmente en el IAPH sobre un cambio de escala hacia lo local mediante la identificación y caracterización de paisajes que integren una carga patrimonial importante y una coherencia narrativa con los paisajes de la demarcación de paisaje cultural en la que se localicen. En este proyecto -Registro de Paisajes de Interés Cultural de Andalucia- se han seleccionado más de 150 paisajes y su documentación se acompaña de una colección de mapas de diversa aproximación temática al paisaje. Pueden citarse: desde el mapa de sintesis de protección patrimonial del territorio en cada paisaje, hasta el mapa representativo de la cobertura superficial en el ámbito de estudio desde lecturas de ortofoto o el de la cuenca visual y grados de visualización en el entorno hacia el área de estudio generado desde modelos digitales de elevaciones.

Paralelamente a estos estudios con carga de análisis de campo y documental, se está desarrollando desde 2008 el proyecto TRAZA enfocado a la sistematización de cartografía digital de los paisajes culturales. El objetivo es recoger toda la información generada en los proyectos de caracterización y de registro de paisajes en una estructura de base de datos asociada a SIG mediante el uso de entidades de tipo zonal, lineal y puntual creando una leyenda cartográfica estructurada y homogénea para su uso con entidades de paisaje y de patrimonio histórico.

Se trata todavía de cartografías para un uso descriptivo de cada ámbito, pero sin duda son la base para la experimentación posterior sobre futuros mapas de paisaje cultural en que puedan reflejarse zonificaciones en tanto a variables o indicadores de calidad, sensibilidad, fragilidad, legibilidad, sociabilidad, identidad, etc., que conduzcan a metodologías para generar delimitaciones coherentes de protección desde el punto de vista de la gestión administrativa. Por otro lado, se estará dando respuesta, tanto a las nuevas recomendaciones del Convenio Europeo del Paisaje en cuanto a una identificación y registro de los paisajes con criterios homogéneos y de calidad, como al reto de avanzar en la aplicación de figuras de protección existentes en nuestra legislación cultural -como son las zonas patrimoniales- desde la vertiente de sus valores paisajísticos. 


\section{El primer mapa geológico parcial de}

Andalucía: se realiza en 1834 por el ingeniero de Minas Frédéric le Play. Comprende el norte de la provincia de Huelva: Estramadure et Nord de I'Andalousie : Essai pour servir au tracé d'une Carte Géologique de l'Estramadure, et de plusieurs Districts des Provinces limitrophea [sic] / Par F. le Play, Ingéniéur des Mines. Biblioteca del Instituto Geológico y Minero de España (Madrid). II/13-1-1 GEO-EXT-1

\section{Bibliografía}

ATLAS de Andalucia, Tomo II: Cartografia ambiental. Sevilla: Consejeria de Obras Públicas y Transportes, 2005

BERQUE, A. (1984) Paysage-empreinte, paysage-matrice: éléments de problématique pour une Géographie Culturelle. L'Espace Géographique, v. 19, 1984, pp. 33-34

BRUNET-VINCK, V. (2004) Méthode pour les atlas de paysages; enseignements méthodologiques de 10 ans de travaux [en línea]. s.l: Ministère de l'Ecologie et du Développement Durables, $2004<$ http://www.developpement-durable.gouv.fr/ IMG/MethodeAtlasPaysages-2004(1).pdf> [consulta: 07/12/10]

CARRERA DíAZ, G. (2009) Atlas del Patrimonio Inmaterial de Andalucia. Puntos de partida, objetivos y criterios técnicos y metodológicos. PH: Boletín del Instituto Andaluz del Patrimonio Histórico, n.o 71, 2009, pp. 18-41

CHAPIN, M.; THRELKELD, B. (2008) Mapping Indigenous Lands. A Practical Guidebook [en línea]. s.l: Center for the Support of Native Lands.Environmental Law Institute, 2008 <http://www.elistore.org/Data/products/d18_23.pdf> [consulta: 07/11/2010]

CHICLANA RODRígUEZ, M. (2005) Representación cartográfica de los paisajes. Memoria final de la beca de investigación Territorio y Paisaje. Centro de Documentación-Alianzas para la Conservación. IAPH (documento interno inédito)

CHICLANA RODRígUEZ, M.; FERNÁNDEZ CACHO, S. (2005) Torres de vigilancia costera y Paisaje: integración de valores culturales y naturales en el Paraje Natural de Maro-Cerro Gordo. PH: Boletín del Instituto Andaluz del Patrimonio Histórico, n. ${ }^{\circ}$ 55, 2005, pp. 93-101

CLARK, J.; DARLINGTON, J.; FAIRCLOUGH, G. (2004) Using Historic Landscape Characterisation [en línea]. s.l: English Heritage\&Lancashire County Council, $2004<$ http://landscapecharacter.org.uk/files/u1/Using-historic-landscapecharacterisation.pdf> [consulta: 07/12/10]

CLAVAL, P. (1999) Los fundamentos actuales de la geografia cultural. Documents d'anàlisi geogràfica, n. ${ }^{\circ} 34,1999$, pp. 25-40

COSGROVE, D.; DANIELS, S. (1988) (ed.) The Iconography of Landscape: essays on the symbolic representation, design and use of past environments. Cambridge: Cambridge University Press, 1988

EXPERIENCING Landscapes: Capturing the 'cultural services' and 'experiential qualities' of landscape. Study Report. July 2009 [en línea]. s.I: Natural England, 2009 <http://naturalengland.etraderstores.com/NECR024> [consulta: 07/12/10] FERNÁNDEZ CACHO, S.; FERNÁNDEZ SALINAS, V.; HERNÁNDEZ LEÓN, E. et ál. (2008) Caracterización patrimonial del mapa de paisajes de Andalucia. $P H$ : Boletín del Instituto Andaluz del Patrimonio Histórico, n. ${ }^{\circ} 66,2008$, pp. 16-31 FERNÁNDEZ CACHO, S.; FERNÁNDEZ SALINAS, V.; HERNÁNDEZ LEÓN, E. et ál. (2010) Paisajes y patrimonio cultural en Andalucía. Tiempo, usos e imágenes. Sevilla: Instituto Andaluz del Patrimonio Histórico, Consejería de Cultura, 2010 (PH Cuadernos, n. ${ }^{\circ}$ 27)

GALINDO GONZÁLEZ, J.; SABATÉ BEL, J. (2009) El valor estructurante del patrimonio en la transformación del territorio. Apuntes, v. 22, n. ${ }^{\circ}$, pp. 20-33 GOFALU am dirweddau hanesyddol = Caring for historic landscapes [en línea] Caerdydd: CADW, 2007 <http://www.cadw.wales.gov.uk/upload/resourcepool/
Caring\%20for\%20Historic\%20Landscapes4584.pdf> [consulta: 07/12/10] HARLEY, J.B. (1988) Maps, Knowledge, and Power. En COSGROVE, D.; DANIELS, S. (ed.) The Iconography of Landscape: essays on the symbolic representation, design and use of past environments. Cambridge: Cambridge University Press, 1988, pp. 277-312

HARLEY, J. B. (1989) Deconstructing the map. Cartographica, v. 26, n. 2 , 1989, pp. 1-20

HERLIHY, P.; DOBSON, J.; AGUILAR, M. et ál. (2008) The AGS Bowman

Expeditions Prototype: Digital Geography of Indigenous Mexico. Final Report [en linea]. Mexican Property Development Survey / Mexico Open Source Geographic Information Systems to Foreign Military Studies Office (FMSO) NRCCLeavenworth, $2008<$ http://web.ku.edu/ mexind/FMSO_Final_Report_2008_ web_version.pdf> [consulta: 07/12/10]

HIRT, I. (2006) ¿Para qué "construir irreversibilidades"? La reconstrucción de Chodoy Lof Mapu, una experiencia autónoma de cartografia mapuche en el sur de Chile. En 52 Congreso Internacional de Americanistas, Sevilla 2006. Reconstrucción de territorios indigenas en América: balances, contiendas y desafios para el siglo XXI [en línea] <http://www.observatorio.cl/contenidos/ naveg/doc7/Sevillal_HIRT.pdf> [consulta: 07/12/10]

LANDSCAPE Character Assessment: Guidance for England and Scotland [en linea] Gloucestershire: The Countryside Agency: Edinburgh: Scottish Natural Heritage, 2002<http://landscapecharacter.org.uk/files/pdfs/LCA-Guidance.pdf> [consulta: 07/12/10]

LEFEBVRE, H. (1991) The Production of Space. Oxford: Blackwell, 1991 LUGINBÜHL, Y. (1994) Méthode pour des atlas de paysages, identification et qualification [en línea]. Paris: Direction de l'Architecture et de I'Urbanisme, Ministère de l'Equipement, 1994 <http://www.developpement-durable.gouv.fr/ IMG/DGALN_MethodeAtlasPaysages-1994_annexes_(1).pdf> [consulta: 07/12/10] NOGUÉ, J.; SALA, P. (2006) Prototipus de catàleg de paisatge. Bases conceptuals, metodológiques i procedimentals per elaborar els catàlegs de paisatge de Catalunya. Document de referència per als grups de treball (edició revisada) [en línea]. Olot i Barcelona: Observatori del Paisatge, $2006<$ https:// docs.google.com/viewer?url=http://www.catpaisatge.net/fitxers/Prototipus2006. pdf> [consulta: 07/12/10]

NOGUÉ, J.; SALA, P. (coord.) (2010) Terres de l'Ebre. Catàleg de Paisatge. Memória. Aprovació definitiva Juliol 2010 [en línea]. Olot: Observatori del Paisatge, $2010<$ http://www.catpaisatge.net/cat/cataleg_presentats_te.php> [consulta: 07/12/10]

QUINTERO MORÓN, V. (2003) El patrimonio inmaterial: ¿intangible? Reflexiones en torno a la documentación del patrimonio oral e inmaterial. En QUINTERO, V.; HERNANDEZ, E. (coord.) (2003) Antropologia y Patrimonio: investigación, documentación y difusión. Granada: Instituto Andaluz de Patrimonio Histórico, Consejeria de Cultura, 2003, pp. 144-157 (Cuadernos Técnicos; n. ${ }^{\circ}$ ) ROCHE, A. (2007) Les unités et structures paysagères dans les Atlas de paysages. Gestion des milieux naturels et biodiversité [en línea]. Ministère de l'Écologie, du Développement et de l'Aménagement Durables <http://www.developpementdurable.gouv.fr/IMG/DGALN_Atlas_unites_structures_paysageres_A_Roche-2007. pdf> [consulta: 07/12/10]

ROCHE, A. (2009) Eléments pour la réalisation et l'actualisation des Atlas de paysages [en línea]. s.l: Ministère de l'écologie, de l'énergie, du Développement durable et de la Mer <http://www.developpement-durable.gouv.fr/IMG/DGALN_ Methodo-realisationactualisationAP.pdf> [consulta: 07/12/10] SEGUIN, J. F. (2009) Identificació i qualificació dels paisatges: I'experiència francesa dels atles del paisatge. En NOGUÉ, J.; PUIGBERT, L.; BRETCHA, G. (ed.) (2009) Ordenació i gestió del paisatge a Europa [en línea]. Olot: Observatori del Paisatge de Catalunya (Plecs de Paisatge; Eines; 2) <http://www.catpaisatge.net/ fitxers/publicacions/ordenacio/Eines\%202_Ordenacio.pdf> [consulta: 07/12/10] SLETTO, B. I. (2009) "We Drew What We Imagined": Participatory Mapping, Performance, and the Arts of Landscape Making. Current Anthropology [en línea], v. 50, n. ${ }^{\circ} 4,2009$, pp. 443-476 <http://soa.utexas.edu/people/docs/sletto/ Sletto_CA_August_2009.pdf $>$ [consulta: 07/12/10] VESCHAMBRE, V. (2009) Lire le paysage dans les atlas français: quelques éléments de réflexion [en línea]. Projets de Paysage <http://www. projetsdepaysage.fr/editpdf.php?texte $=514>$ [consulta: 07/12/10] WU, C. J.; ISAKSSON, K. (2008) Participatory mapping as a tool for capturing local perspectives on cultural landscape-case study of Ostlänken [en linea]. Mistra. Sweden <http://www.mistra.org/download/18.87749a811cbd4c4 fb4800010902/Participatory+mapping+as+a+tool.pdf> [consulta: 07/12/10] 